\title{
Preconception and prenatal testing of biologic fathers for carrier status
}

Beth A. Pletcher, $M D^{1,2}$, and Maureen Bocian, $M D^{1,3}$

Key Words: Carrier screening, genetic testing, reproductive genetics, coverage policy

Disclaimer: This guideline is designed primarily as an educational resource for medical geneticists and other health care providers to help them provide quality medical genetic services. Adherence to this guideline does not necessarily assure a successful medical outcome. This guideline should not be considered inclusive of all proper procedures and tests or exclusive of other procedures and tests that are reasonably directed to obtaining the same results. In determining the propriety of any specific procedure or test, the geneticist should apply his or her own professional judgment to the specific clinical circumstances presented by the individual patient or specimen. It may be prudent, however, to document in the patient's record the rationale for any significant deviation from this guideline.

The familial nature of genetic conditions often requires the testing of parents and other family members in order to determine the relationship of a genetic change to a clinical phenotype or to determine potential reproductive risks. When required as part of prenatal and preconceptional genetic testing services, time constraints and the costs and risks of alternatives to testing parents require that payers have established policies for how both maternal and paternal tests that inform fetal testing choices or the interpretation of fetal testing results will be covered.

\section{INTRODUCTION}

Genetic testing, in the context of a planned or ongoing pregnancy, frequently poses special challenges to health care providers. Barriers to such testing may include: time constraints, labor intensive laboratory procedures, the need to test more than one family member, and/or inability of uninsured biologic fathers to be tested, despite the fact that such testing is being done for fetal diagnosis. As more and more genetic tests are becoming standard of care for women who are, or may become pregnant, we need to consider how best to provide appropriate and efficient care for these patients.

\section{ISSUES}

- Problems are often encountered in the testing of a fetus or pregnancy for genetic conditions when the mother is identified to be a carrier of a genetic condition, and the

\footnotetext{
For the ${ }^{1}$ ACMG Professional Practice and Guidelines Committee, ${ }^{2}$ Doctors Office Center, Newark, NJ, ${ }^{3}$ University of California Irvine Medical Center, Orange, CA

biologic father has neither insurance coverage nor the resources to pay for carrier testing.

- If the father is unable to be screened, pregnant women and their fetuses may undergo unnecessary invasive procedures, such as CVS or amniocentesis, that are also considerably more expensive than carrier testing, in order to determine if the fetus is affected.

- Carrier testing of prospective parents should be undertaken in the safest and most cost effective way.

- Pregnancy itself imposes time limits for carrier testing which also must be taken into consideration.

\section{CONDITIONS TO BE TESTED FOR}

Some common conditions (there are many not listed here)

- Carrier status for genetic conditions that are found frequently in specific populations

- Hemoglobinopathies (sickle cell trait, C trait, thalassemia trait, hemoglobin E)

- Cystic fibrosis

- Tay-Sachs disease

- Canavan disease

\section{Special circumstances}

- Cytogenetic studies in the context of a suggestive family history (i.e., recurrent pregnancy loss, a fetal cytogenetic abnormality such as a chromosomal translocation, individual with a known chromosomal rearrangement, individual with multiple anomalies \pm mental retardation). (Depending on the specific family history, cytogenetic testing of only the male partner may be the appropriate screening test.)

- Previous child or other family history of an individual with a specific genetic disorder that is amenable to prenatal diagnosis. (For some less common disorders in this 
category, preconception screening may be required in order to permit adequate time for follow-up testing or complex laboratory investigations, and to allow for the more cost effective sequential, rather than simultaneous testing.)

\section{DISCUSSION}

As more carrier screening tests are offered to women in the context of a pregnancy at-risk for a genetic disorder, a mechanism must be in place to provide timely and accurate diagnosis for the lowest cost and with the fewest risks to the pregnant woman and her developing fetus. Enabling biologic fathers to be tested in order to adequately assess risk for an ongoing or planned pregnancy is the first step toward attaining this goal. Since fetal testing may be covered by the maternal insurance plan, it is reasonable to expect that paternal testing for fetal diagnosis would be reimbursed by the same insurer. From a patient care perspective, the best time to perform carrier screening is prior to a recognized pregnancy, as it allows couple to consider other reproductive options such as preimplantation genetic diagnosis, use of donor gametes, or adoption. Yet, many patients are not covered for such testing until a pregnancy is underway. Whereas some laboratories perform concurrent testing on expectant couples for fetal conditions (i.e., uniparental disomy) and are able to bill the insurer on a single invoice and generate a single laboratory report, the vast majority of fetal diagnoses involving samples from multiple individuals result in multiple invoices and multiple reports. Sequential testing of potential carrier couples may be even more problematic in this regard, but certainly these obstacles are not insurmountable. Given the advances in genetic health care and availability of preconceptional and prenatal carrier tests, the time is at hand for health insurance carriers to come together with clinical and laboratory health care providers to ensure equitable coverage for fetal testing that optimizes care for patients across the US.

\section{RECOMMENDATIONS}

In light of the above, optimal care for a couple potentially at-risk for a diagnosable genetic disorder in their fetus requires that:

- Women be offered appropriate screening for carrier status prior to conception. If this is not possible, then the expectant mother should be screened as early as possible in the prenatal period.

- If a woman is found to be a carrier of an autosomal recessive disorder, then the biologic father should be offered carrier testing to assess the risk to their offspring.

- When a woman is tested at a more advanced gestational age (i.e., after 14 weeks), she and the biologic father should be tested concurrently rather than sequentially because of time constraints.

- Based on a significant paternal family history, testing of the biologic father alone or initially may be recommended.

- When testing of the biologic father is indicated, such testing should be covered as part of global obstetrical care. 